\title{
ESTUDIOS CROMOSOMICOS EN TURNERA Y PIRIQUETA (TURNERACEAE)
}

\author{
por AVELIANO FERNANDEZ*
}

\section{Summary}

The chromosome number from 90 populations of 19 species and 5 subspecies of Turnera and 7 species of Piriqueta are reported for the first time. Previous reports of 6 tax a of Turnera and one of Piriqueta are confirmed.

Six of the 9 series of the genus Turnera were studies cytologically. Four of the six has $\mathrm{x}=7$ chromosomes (Salicifoliae, Stenodictyae, Leiocarpae and Mycrophyllae). Canaligerae has $\mathrm{x}=5$ and Papilliferae posesses $\mathrm{x}=13$ found in the only species of the series $T$. chamaedryfolia with $2 n=26$. It is suggested that the most primitive basic number is $x=7$ which gave rise to $x=5$ and $x=13$.

Autopolyploids and alopolyploids played an important role in the evolution of the species of the Turnera and Piriqueta. Among the known species of Turnera with basic number $\mathrm{x}=7$, $40 \%$ are polyploids; one of them, T. sidoides with 5 subspecies, has only 2 diploids among 20 populations, the others are autotetraploids, autohexaploids and one autooctoploids. In the series Canaligerae with basic number $\mathrm{x}=5$, only $34 \%$ of the species are diploids, $33 \%$ is represented by diploid and tetraploid cytotypes and the remaining $33 \%$ are alopolyploids. Different degress of diploidization were found in the different autopolyploids. In the genus Piriqueta with basic number $\mathrm{x}=7,27 \%$ of the species are polyploids.

* Miembro de la Carrera del Investigador - CONICET. Instituto de Botánica del Nordeste (UNNE-CONICET). C.C. 209 -3400 Corrientes, Argentina. 


\section{Introducción}

Urban (1883) en su monografía reconoció 5 géneros en Turneraceae y actualmente esta familia cuenta con 10 géneros (Arbo comunicación personal) con aproximadamente 170 especies. El género Turnera con alrededor de 100 especies americanas, distribuidas desde el sur de EEUU hasta Argentina y con 2 especies africanas; Piriqueta con alrededor de 30 especies distribuidas también desde el sur de EEUU hasta Argentina y con una especie africana. Estos dos géneros representan el $80 \%$ de la familia. Los demás géneros son africanos a excepción de Adenoa monotípico y endémico de Cuba (Arbo, 1977).

De las 170 especies de Turneraceae, se conocía el número cromosómico de 6 táxones de Turnera y 4 especies de Piriqueta. Los 6 táxones de Turnera son: T. ulmifolia var. angustifolia $2 \mathrm{n}=30$ (Hamel, 1965; Barrett, 1978), var. intermedia $2 \mathrm{n}=10$ y 20 , var. surinamensis $2 \mathrm{n}=10$, var.grandiflora $2 \mathrm{n}=10$, var, orientalis $2 \mathrm{n}=30$ (Barrett y Shore, 1980). La var. elegans se conocía únicamente con 2n=20 (Raman y Kesavan, 1964; Barrett, 1978); recientemente Arbo y Fernández (1983) encontraron poblaciones tetraploides, $2 n=20$ y diploides, $2 n=10$. Estos últimos autores reconocen las variedades de $T$. ulmifolia como especies independientes por presentar una combinación característica de rasgos morfológicos diferenciales (Arbo y Fernández, 1983; Arbo, 1985).

Las 4 especies de Pirqueta que fueron estudiadas cariológicamente son: $P$. glabrescens, $P$. tomentosa (Lewis et al., 1962), $P$. cistoides y $P$. caroliniana (Ornduff, 1970), todas ellas poseen $2 \mathrm{n}=14$.

En el presente trabajo se da a conocer el número cromosómico de 19 especies de Turnera y 5 subespecies de $T$. sidoides y de 7 especies de Piriqueta. Se confirma el número cromosómico de los 6 táxones de Turnera anteriormente contados y el de $P$. cistoides. Se discute la importancia taxonómica del nivel de ploidía y del comportamiento de los cromosomas durante la meiosis en Turnera, como también el rol de la poliploidía en la evolución de las especies de Turnera y Piriqueta. 
Material y métodos

La procedencia de las especies estudiadas figura en el cuadro I y en su mayoría están cultivadas en el Jardín del Instituto de Botánica del Nordeste (IBONE). Las semillas utilizadas para efectuar los preparados para los estudios mitóticos, fueron extraídas por M.M. Arbo de ejemplares de herbario que se encuentran depositados en el Herbario del IBONE, CTES. Una vez cortadas las puntas de las raíces de las semillas germinadas, éstas fueron transferidas a macetas y de las plantas obtenidas fueron tomados los botones florales para los estudios meióticos.

Los estudios mitóticos fueron realizados en puntas de raíces, las que fueron pretratadas con 8-oxiquinoleína $(0,002 \mathrm{M})$ durante tres horas a temperatura de laboratorio y los estudios meióticos en botones florales. En ambos casos el material fue fijado en etanol absoluto y ácido láctico (5:1) toda la noche, en refrigerador; conservado en etanol $70^{\circ}$ durante tres a cinco días, en refrigerador y coloreado con la técnica de Feulgen que consiste en: a) hidrólisis en $\mathrm{ClH} 1 \mathrm{~N} \mathrm{a} 60^{\circ}$ durante ocho minutos, b) coloración en reactivo de Schiff, c) aplastado en orceína lacto-acética modificada (Mroginski y Fernández, 1979).

La fertilidad de polen se estimó coloreándolo con carmínglicerina, contando no menos de 700 granos de polen.

Resultados y discusión

Turnera

En el cuadro I se indican los números cromosómicos de las 25 especies de Turnera y 8 especies de Piriqueta estudiadas.

En la serie Salicifoliae se estudió una especie, T. Weddellia$n a$ con $2 \mathrm{n}=14$ (Fig. 1) y en la serie Stenodictyae se estudió $T$. macro phylla también con $2 \mathrm{n}=14$.

A la serie Leiocarpae pertenecen las siguientes especies di- 


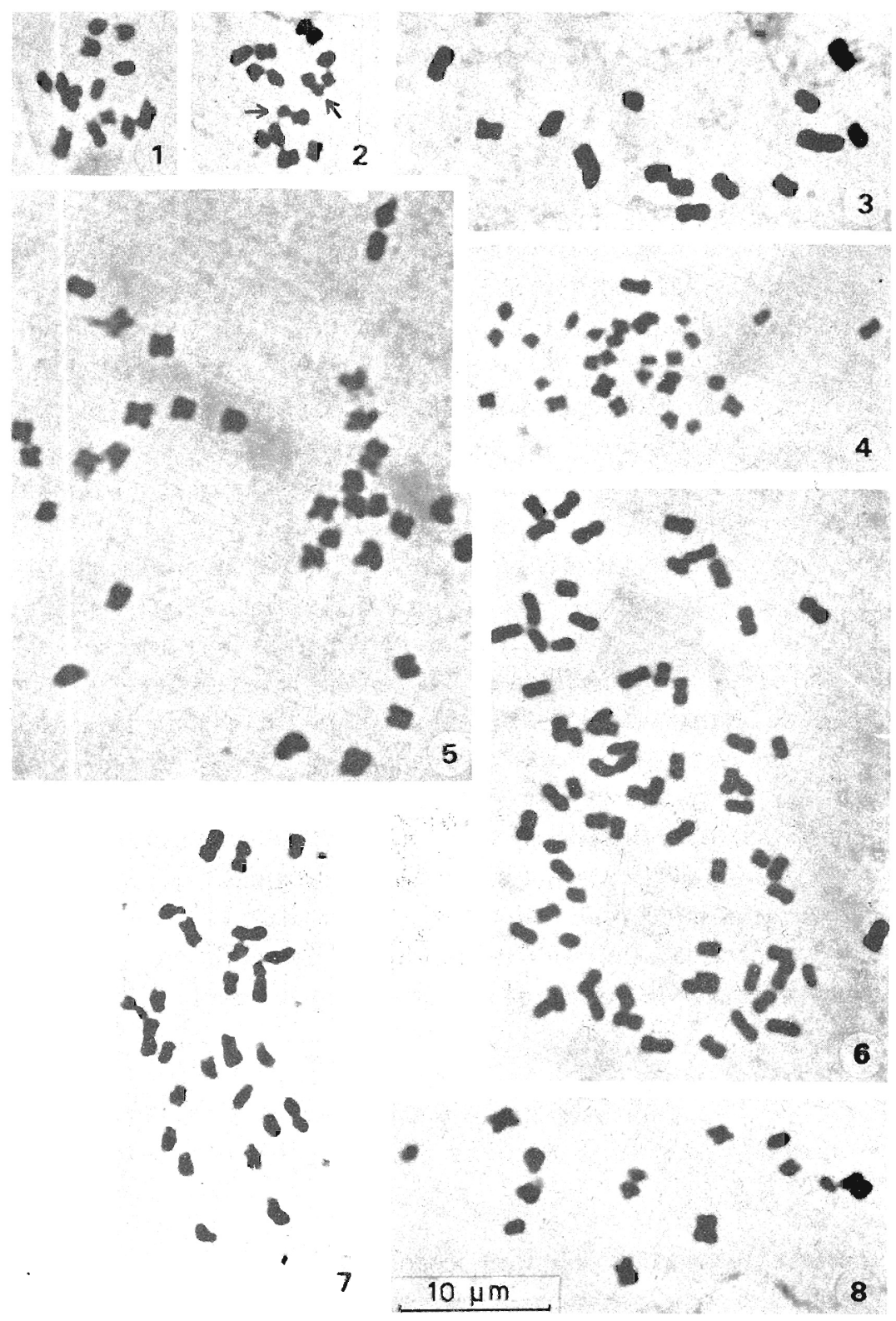

Fig. 1-8. Cromosomas mitóticos. 1, Tumera Weddelliana, $2 \mathrm{n}=14 ; 2, T$. pumilea, $2 \mathrm{n}=$ 14, las flechas indican cromosomas con satélite; 3, T. Hassleriana, citotipo diploide, $2 \mathrm{n}=14 ; 4, T$. Hassleriana, citotipo tetraploide, $2 \mathrm{n}=28 ; 5$, T. sidoides ssp. sidoides, $2 \mathrm{n}$ $=32 ; 6, T$. opifera, $2 \mathrm{n}_{\mathbf{z}} 70 ; 7, T$. chamaedryfolia, $2 \mathrm{n}=26 ; 8$, Piriqueto aff. duarteana, $2 n=14$. 
Cuadro I. Números cromosómicos, coleccionista, números de herbario y procedencia del material

\begin{tabular}{llll}
\hline Especie & $2 \mathrm{n}$ & Fig. & Coleccionista* y procedencia \\
\hline
\end{tabular}

\section{Tumera}

Serie Salicifoliae

T. Weddelliana Urban \& Rolfe 14

Serie Stenodictyae

T. macro phylla Urban

\section{Serie Leiocarpae}

T.nervosa Urban

T. pumilea L.

,

T. melochioides Camb.

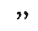

T. Hassleriana Urban

T. lamiifolia Camb.

T. sidoides L. ssp. sidoides

$$
32,34,39
$$

1

S.21213. Paraguay, dep. Chaco, Colonia San Alfredo.

Cid 2452, Brasil, PA, rio Trombetas, $5 \mathrm{~km}$ da cidade de Oriximiná.

A.2076, Argentina, Ctes., dep. Ituzaingó, arroyo Itaembé.

2 A.2110, Argentina, Ctes. dep. Ituzaingó, Villa Olivari.

K.37189, Brasil, Maranhao, Timon, $7 \mathrm{~km}$ del puente a Teresina.

K.36653, Bolivia, dep. S. Cruz, $10 \mathrm{~km}$ S de San José de Chiquitos. K.38621, Brasil, PI, Floriano.

K.38695, Brasil, BA, $65 \mathrm{~km}$ W de Barreiras.

K.38754, Brasil, PI, $7 \mathrm{~km}$ E de Nazaré.

3 K.36444, Bolivia, dep. S. Cruz, $8 \mathrm{~km} \mathrm{~W}$ de Santiago.

A.1735, Paraguay, dep. Paraguarí, Salto del Chololó.

4 Vanni 351, Paraguay, dep. Concepción.

K.33168, Brasil, Brasilia, Parque Nacional.

A.2497, Uruguay, dep. Lavalleja, Cerro Arequita, $12 \mathrm{~km} \mathrm{~N}$ de Minas.

5 A.2435, Brasil, RS, Alegrete, BR290.

* Las siguientes iniciales significan: A.: Arbo, K.: Krapovickas y S.: Schinini. 


\section{Cuadro I (continuación)}

\begin{tabular}{llll}
\hline Especie & $2 \mathrm{n}$ & Fig. & Coleccionista y procedencia \\
\hline
\end{tabular}

T. sidoides L.ssp. carnea

(Camb.) Arbo

,"

T. sidoides L. ssp. integrifolia

(Griseb.) Arbo

,"

T. sidoides L. ssp. holosericea

(Urban) Arbo

",

T. sidoides L. ssp. pinnatifida (Juss. ex Poir.) Arbo

T.opifera Mart.

70

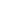

8

8

8

8

2

2

8

28

S.21 711, Argentina, Ctes. dep. Mercedes.

A.2510, Uruguay, dep. Tacuarembó, Cerro de las Minas.

Cristóbal 1782, Argentina, Ctes. dep. Ituzaingó, $10 \mathrm{~km} \mathrm{~W}$ de Colonia Liebig.

K.25847, Argentina, Ctes., dep. Santo Tomé, Garruchos.

A.385, Argentina, Ctes. dep. Concepción, Tabay.

A.241 8, Argentina, Ctes. dep. P. de los Libres.

A.2506, Uruguay, dep. Durazno, $35 \mathrm{~km} \mathrm{~N}$ de Durazno, ruta 5.

A.2421, Brasil, RS, $14 \mathrm{~km} \mathrm{~W} \mathrm{de}$ Alegrete.

A.2487, Brasil, RS, $50 \mathrm{~km} \mathrm{~S} \mathrm{de}$ Bajé.

A.2419, Brasil, RS, $6 \mathrm{~km} \mathrm{E}$ de Uruguayana.

A.2525, Uruguay, dep. Artigas, 64 $\mathrm{km}$ SE de Artigas, ruta 30.

A.2461, Brasil, RS, $70 \mathrm{~km} \mathrm{E} \mathrm{de}$ S. Gabriel.

A.2513, Uruguay, dep. Tacuarembó, $53 \mathrm{~km}$ SSW de Tacuarembó.

A.2478, Brasil, RS, $27 \mathrm{~km} \mathrm{~S}$ de Caçapava do S. BR-1 53 .

A.2494, Uruguay dep. Cerro Largo, $15 \mathrm{~km} \mathrm{~S}$ de Melo, ruta 8.

A.2495, Uruguay, dep. Treinta y Tres, $40 \mathrm{~km} \mathrm{~N}$ de Treinta y Tres. S.21648, Argentina, Ctes. dep. Curuzú Cuatiá, arroyo Punta.

A.2411, Argentina, Ctes. dep. Capital, Molina Punta.

Fernández 370, Cult. Ctes. Brasil, MG, Grão Mogol. 


\section{Cuadro I (continuación)}

\begin{tabular}{llll}
\hline Especie & $2 n$ & Fig. & Coleccionista y procedencia \\
\hline
\end{tabular}

Serie Microphyllae

T. diffusa Willd.

Serie Papilliferae

T. chamaedryfolia Camb.

26

$"$

26

Serie Canaligerae

T. caerulea DC.

,

10

T. surinamensis (Urban)

10

Arbo, nov. comb. ined.

T. scabra Millspaugh ( $=$ T. ulmifolia var.intermedia)

n

T. concinna Arbo

T. Krapovickasii Arbo
K.38071, PE, Mun. Bu ique, Cajueiro.

Carvalho 538, Brasil, BA, Conceiçao de Feira.

7 Noblick 3175, Brasil, BA, Feira de Santana.

K.38740, Brasil, PI. Bom Jesus, BR-135.

K.37914, Brasil, GO, BR--153, 18 $\mathrm{km} \mathrm{N}$ de Colina.

K.38751, Brasil, PI, Floriano.

Barrett 1126 , Venczuela, Calabozo, Guarico.

Araquistain 1354, Nicaragua, dep. Managua, Península de Chiltepe, 9 $\mathrm{km}$ SE de Matearc.

Jiménez 8769, Rep. Dominicana, prov. de la Vega, Loma de! Puerto. Varni 213, Paraguay, dep. S. Pedro, $31 \mathrm{~km}$ de Colonia Guayaibí, ruta 3 .

K. 38858, Bolivia, dep. Tarija, prov. O'Connor, $3 \mathrm{~km} \mathrm{~S}$ de Entre Ríos.

K.39177, Bolivia, dep. Tarija, prov. Gran Chaco, Villa Niontes.

K. 39099, Bolivia, dep. Tarija, prov. O'Connor, $19 \mathrm{~km}$ E de Entre Ríos. Ahumada 4549, Argentina, Jujuy, ruta 34 y Puente río Negro III.

K.36346, Bolivia, dep. S. Cruz, prov. Chiquitos, Roboré.

Beck 9433, Paraguay, dep. Nucva Asunción. Grl. E.A. Garay $13 \mathrm{~km}$ hacia Estigarribia.

S. 19514, Argentina, Salta, dep. Anta, El Quebrachal. 


\section{Cuadro I (continuación)}

\begin{tabular}{llll}
\hline Especie & $2 \mathrm{n}$ & Fig. & Coleccionista \\
\hline
\end{tabular}

T.grandiflora (Urban) Arbo 10

,

,

,

》

,

T. hermannioides Camb.

T. aff. coriacea Urban

T. subulata Smith

T. grandidentata (Urban) Arbo 20

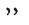

T. angustifolia Miller

T.orientalis (Urban) Arbo

T. ulmifolia L. sensu stricto

T. Aurelii Arbo
K.37471, Paraguay, dep. Villa Hayes, km 249, ruta Trans Chaco.

K 34262, Paraguay, dep. S. Pedro, 35 $\mathrm{km} N$ de San Estanislao.

A. 386, Argentina, Ctes. dep. Capital, Cambá Punta.

A.1736, Paraguay, dep. Paraguarí, Saltos del Chololó.

Fernández 366, Argentina, Ctes. dep. San Cosme, Paso de la Patria.

Fernández 367, Argentina, Ctes. dep. Empedrado, El Sombrerito, INTA.

S.21385, Brasil, MT, do Sul, Bela Vista.

K. 38729, Brasil, PI, mun. Corrente. K. 38793, Brasil, BA, $29 \mathrm{~km} \mathrm{~S}$ de Juazeiro.

K. 37115 , Brasil, Amazonas, Manaus. A.1674, Paraguay, dep. Central, margen sur del río Salado.

A.1774, Paraguay, dep. Paraguarí, Saltos del Chololó.

S.23981, Paraguay, dep. Cordillera, Cordillera de Altos, Col. Tobaty. Barrett 1254, Costa Rica, prov. Guanacaste, Ca ñas.

A.2121, Argentina, Ctes. dep. Ituzaingó, Villa Olivari ruta 12.

A.1538, Argentina, Ctes. dep. Capital, puerto Italia.

A.1497, Argentina, Ctes. dep. Capital, Punta Arazá.

Cabral 358, Argentina, Misiones, dep. San Ignacio, Teyú Cuaré.

A.2698, cult. Ctes. semillas procedentes de Miami, Florida, U.S.A.

S.23860, cult. Ctes, semillas procedentes de Paraguay, dep. Cordillera, río Salado, camino a Emboscada. 
Cuadro I (continuación)

\begin{tabular}{llll}
\hline Especie & $2 \mathrm{n}$ & Fig. & Coleccionista \\
\hline
\end{tabular}

\section{Piriqueta}

P. cistoides (L.) Griseb.

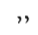

,

,

,

P. viscosa Griseb.

P. aff. duarteana Urban

P. serrulata Urban

P. racemosa (Jacq.) Sweet

P.rosea (Camb.) Urban var. occidentalis Urban

P. suborbicular is (St. Hil.)

Arbo

P. taubatensis (Urban) Arbo
14

14

14

14

14

14

14

14

14

14

28

28

42

K.33384, Brasil, MG, $35 \mathrm{~km} \mathrm{E}$ de Araxá.

K 35283 , Brasil, SP, Araquara.

K.37136, Brasil, PA, Santarem.

K.37833, Brasil, GO, Guripí BR153.

Jiménez 9081, Rep. Dominicana, prov. de Santiago, Rincón de Piedra.

A.2115, Argentina, Ctes. dep. Ituzangó, Villa Olivari.

Cristóbal 1706, Argentina, Ctes. dep. Capital, $7 \mathrm{~km}$ E de Ctes.

8 K.38735, Brasil, PI, Gilbués.

K.34405, Brasil, MT do Sul, 38 km $W$ de Ribas do Rio Pardo.

K 38807 , Brasil, BA, Baiza Grande.

'K.34428, Brasil, MT do Sul, 85 km de Campo Grande.

A.2479, Brasil, RS, rio Camaquá $70 \mathrm{~km} \mathrm{NE} \mathrm{de} \mathrm{Bajé} \mathrm{BR} \mathrm{-} 153$.

A.2544, Argentina, Misiones, Candelaria.

ploides 2n=14: T. pumilea (Fig. 2), T. melochioides y T. Hassleriana (Fig. 3), esta última especie tiene además dos poblaciones con citotipo tetraploide, $2 n=4 x=28$ (Fig. 4). Otras especies estudiadas de esta serie son: $T$. lamiifolia $2 \mathrm{n}=4 \mathrm{x}=28 ; T$. sidoides, complejo poliploide con cinco subespecies, que presentan diferentes grados de ploidía: ssp. sidoides con $2 n=28$ (Arbo 2497) y $2 n=$ 32 (Fig. 5), 34 y 39 contados en tres semillas provenientes de un mismo especimen (Arbo 2435), probablemente sean segregantes de un pentaploide $2 n=5 x=35$; ssp, carnea con $2 n=14,28$ y 42 es decir di, tetra y hexaploides; ssp. integrifolia con $2 n=14,28$, 42 y 56 di, tetra, hexa y octoploide; ssp. holosericea con $2 n=$ 
28 y 42 ; ssp. pinnatifida con $2 n=28$. La especie estudiada con el nivel más alto de ploidía es $T$. opifera con $2 \mathrm{n}=10 \mathrm{x}=70$ (Fig.6).

T. diffusa es una especie dipioide $(2 n=14)$ de la serie $M i$ crophyllae. Todas las especies mencionadas anteriormente tienen el número básico $\mathrm{x}=7$, mientras que $T$. chamaedryfolia de la serie Papilliferae, presenta $2 n=26$ (Fig. 7), con el número básico $\mathrm{x}=13$.

Las especies pertenecientes a la serie Canaigerae poseen el número básico $\mathrm{x}=5$. Los resultados hallados en $T$. surinamensis, T. caerulea, T. concinna (Arbo, 1986), T. hermannioides y $T$. aff. coriacea determinan que son diploides $(2 n=10)$. Se han detectado ires especies con citotipos diploides $(2 n=10)$ y atraploides $(2 \mathrm{n}=20): T$. subulata (Arbo y Fernández, 1983), T. scabra y T. Krapovickasii. En cambio en T. grandiflora se hallaron citotipos diploides $(2 n=10)$ y octoploides $(2 n=40)$. T. grandidentata es una especie tetraploide $(2 n=20)$, mientras que $T$. angustifolia, $T$. orientalis y $T$. ulmifolia son hexaploides, $2 \mathrm{n}=30$ y $T$. Aurelii, es octoploide, $2 n=40$.

En el género Turnera, se analizó el comportamiento meiótico en 2 series: Leiocarpae y Canaligerae. En la serie Leiocarpae el estudio se intensificó en el complejo $T$. sidoides, se analizó una población diploide, 4 tetraploides y 2 hexaploides (cuadro V). En la población diploide, de Ia ssp. integrifolia, Arbo 385 $(2 n=14)$, el comportamiento niè́tico fue regular formando siempre 7 II (Fig. 15) en las 81 células analizadas. En ia ssp. integrifolia tetraploide $(2 n=28)$, Arbo 2418 , en las 9 células estudiaras había 7 configuraciones diferentes, se encontró por lo menos un teîravalente por célula y hasta un máximo de 6 IV. En la ssp. carnea, Arbo $25: 0(2 n=28)$, se analizaron 84 células, encontrándose 12 configuraciones diferentes, siendo las más frecuentes, células con 8 II +3 IV y células con 6 II +4 IV . En la ssp. holosericea se estudiaron 2 poblaciones tetraploides: en Arbo $2461(2 n=28)$, se analizaron 21 células, encontrándose 7 configuraciones diferentes, siendo la más frecuente 12 II 
+1 IV (38\%) y en Arbo 2525, se encontraron 6 configuraciones diferentes en 8 células analizacias. Las dos poblaciones hexaploides estudiadas perteneceri a la ssp. carnea, en Krapovickas 25847 el $44 \%$ de las células tenía hexavalentes y en Cristóbal 1782 habian hexavalentes (Fig. 17) en $87,5 \%$ de las células y en ambos se hallaron 1-7 IV en todas las células.

Cuadro II. Promedio \pm E.S. y variación de las asociaciones cromosómicas en metafase I, en especies de Turnera (Serie Canaligerae)

\begin{tabular}{|c|c|c|c|c|c|}
\hline Especie & $2 n$ & I & II & IV & N.CMP \\
\hline T. caerulea K. 38740 & 10 & & 5 & & 76 \\
\hline T. aff. coriacea K. 38793 & 10 & & 5 & & 109 \\
\hline T. hermannioides K. 38729 & 10 & & 5 & & 144 \\
\hline T. surinamensis K. 38751 & 10 & & 5 & & 51 \\
\hline \multirow[t]{3}{*}{ T. Krapo vickasii Ah. 4549} & 10 & 0,01 & 4,99 & & 101 \\
\hline & & $\pm 0,01$ & $\pm 0,008$ & & \\
\hline & & $(0-2)$ & $(4-5)$ & & \\
\hline T. grandiflora A. 386 & 10 & & 5 & & 98 \\
\hline T. grandidentata A. 1674 & 20 & & 10 & & 38 \\
\hline T. orientalis A. 1538 & 30 & & 15 & & 26 \\
\hline T. ulmifolia A. 2698 & 30 & & 15 & & 77 \\
\hline \multirow[t]{3}{*}{ T. Aurelii S. 23860} & 40 & & 19,85 & 0,07 & 28 \\
\hline & & & $\pm 0,09 \quad \pm$ & $=0,04$ & \\
\hline & & & $(18-20)$ & $(0-1)$ & \\
\hline
\end{tabular}

Los resultados hallados en el complejo $T$. sidoides, muestran que los tetraploides y hexaploides se comportan citológicamente como au topoliploides. Los tetraploides forman un alto porcentaje de tetravalentes y los hexaploides de hexavalentes. En el octoploide se pudo observar asociaciones cromosómicas hasta octovalentes. Estos autopoliploides presentan diferentes grados de diploidización (cuadro V). De los hexaploides estudiados $(2 \mathrm{n}=$ 
42), que pertenecen a la ssp.carnea, en Krapovickas 25847, la diploidización es mayor que en Cristóbal 1782; en el primero el promedio de bivalentes es 12,3 mientras que en Cristóbal 1782 el promedio es sólo de 7,87. Entre los tetraploides, el grado de diploidización más alto está en Arbo 2461, con un promedio de bivalentes de 10,66 mientras que en Arbo 2510, el promedio es 6,36 .

T. nervosa una especie diploide $(2 \mathrm{n}=14)$ también de la serie Leiocarpae presentabala meiosis regular formando siempre 7 II en todas las células analizadas.

En la serie Canaligerae $(\mathrm{x}=5)$ los diploides $(2 \mathrm{n}=10): T$. caerulea, T. hermannioides, T. aff. coriacea, T. grandiflora (Fig. 9) y $T$. surinamensis presentaban la meiosis regular formando 5 II (cuadro II). El citotipo diploide de T. Krapovickasii presentó 5 II en 100 células y en una sóla 2 I + 4 II; en anafase I se encontró una célula con segregación $4-6$ y 24 células con segregación $5-5$. En el citotipo tetraploide $(2 n=20)$ de $T$. scabra (Fig. 13) y $T$. Krapovickasii siempre se encontraron tetravalentes en todas las células. Sin embargo en $T$. grandidentata, $2 \mathrm{n}=4 \mathrm{x}=$ 20. todas las células forman 10 II (Fig. 10). En T. ulmifolia (Fig. 11) y $T$. orientalis, $2 \mathrm{n}=6 \mathrm{x}=30$, todas las células forman 15 II y en $T$. Aurelii, $2 \mathrm{n}=8 \mathrm{x}=40$, se observaron 20 II (Fig. 12) en 26 células y 18 II +1 IV en 2 células.

Lewis et al. (1951) encontraron 3 especies de Delphinium que poseen citotipos diploides y tetraploides. Dos especies presentaban más poblaciones diploides que tetraploides, en la tercera, el número de poblaciones tetraploides era un poco mayor. El mismo autor (Lewis, 1.967) posteriormente concluye que los diploides y sus derivados autotetraploides constituyen una sóla especie, porque hay continuidad genética entre ellos. Arbo y Fernández (1983), llegan a la misma conclusión al estudiar los citotipos diploides $(2 n=10)$ y tetraploides $(2 n=20)$ y el híbrido triploide $(2 n=15)$ en $T$. subulata. En el presente trabajo se ha estudiado otra población autotetraploide $(\mathrm{K} .37115)$ en $T$. subulata, observándose que el grado de diploidización es mayor en las 

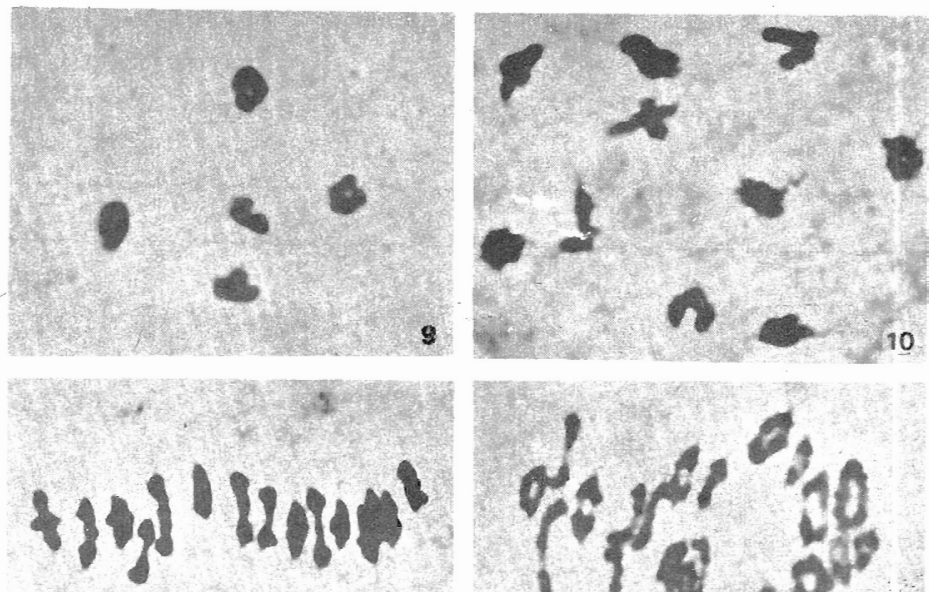

11
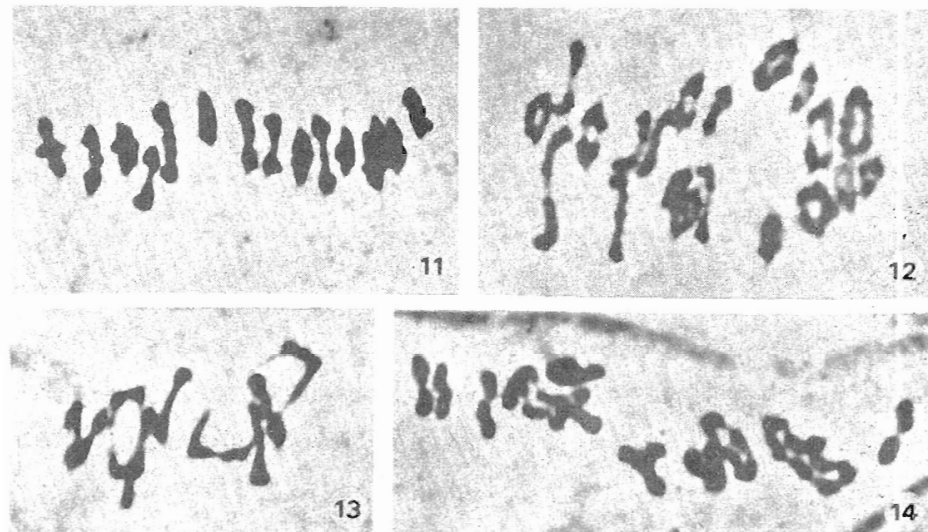

13
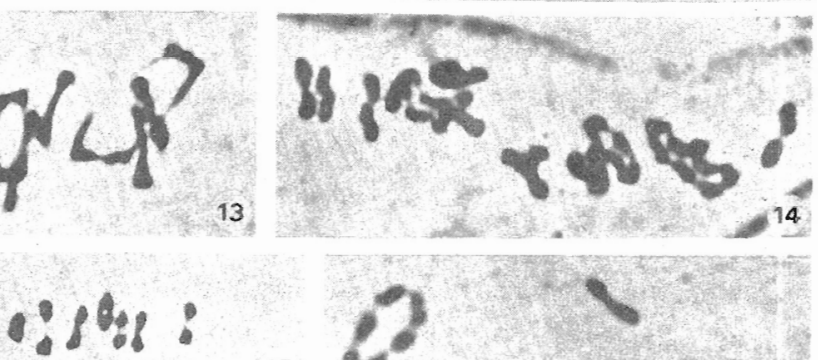

15
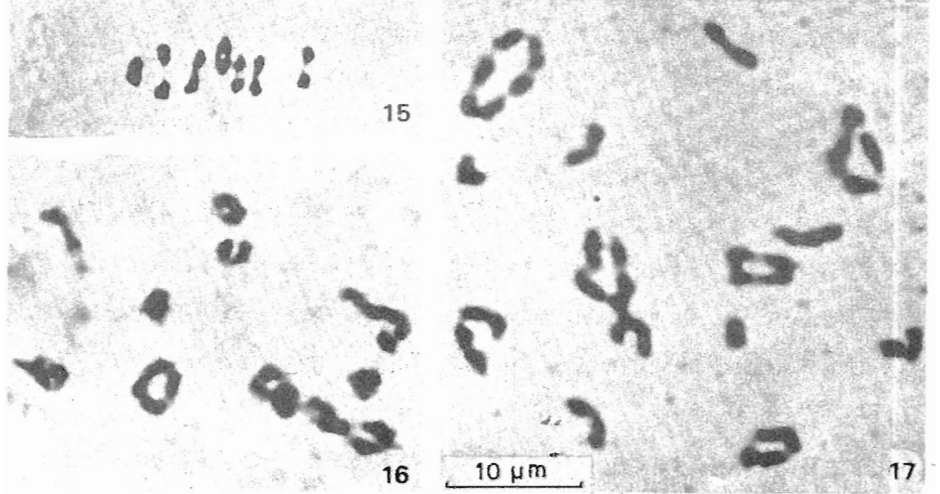

Fig. 9-17. Cromosomas meióticos. 9, Tumera grandiflora, 5 II: 10, T. grandidentata, 10 II: 11, T. ulmifola, 15 II: 12; T. Aurelii, 20 Il; 13, T. scabra citotipo tetra ploide, 5 IV: $14, T$. grandiflora, citotipo octo ploide, 3 VIII + 2 IV + 4 II 1 15, T. sidoides ssp. sidoides $(2 \mathrm{x}), 1$ 7II: $16, T$. sidoides ssp. holosericea $(4 \mathrm{x}), 4 \mathbf{I V}+6$ II: $17, T$. sidoides ssp. carnea $(6 \mathrm{x}), 1 \mathrm{VI}+2 \mathrm{~V}+3 \mathrm{IV}+6 \mathrm{II}+2 \mathrm{I}$. 
Cuadro III. Promedio \pm F.S. y variación de las asociaciones cromosómicas en metafase I, en citotipos tetraploides de 3 especies de Turnera (Serie Canaligerae)

\begin{tabular}{lcccc}
\hline Especie & 2n & II & IV & N.CMP \\
\hline T. scabra J.8769 & 20 & $3,16 \pm 0,71$ & $, 3,41 \pm 0,35$ & 16 \\
& & $(0-8)$ & $(1-5)$ & \\
T. subulata K. 37115 & 20 & $5 \pm 1,06$ & $2,5 \pm 0.53$ & 8 \\
T. Krapovickasii S. 19514 & & $(0-8)$ & $(15)$ & \\
& 20 & $3,37 \pm 0,39$ & $3,31 \pm(0,19$ & 29 \\
& & $(0-8)$ & $(1-5)$ &
\end{tabular}

estudiadas previamente. T. scabra y T. Krapovickasii también poseen citotipos diploides $(2 n=10)$ y tetraploides $(2 n=20)$; las plantas tetraploides presentaban tetravalentes en todas las células analizadas en metafase 1 (cuadro IiI), este comportamiento está indicando que estos citotipos son autot etraploides (Stebbins, 1951). La diploidización en los autotetraploides de estas especies es similar, siendo el promedio de bivalentes de 3,16 y 3,36 respectivamente. La otra especie con dos niveles de ploidía, T. grandiflora, posee citotipo diploide $(2 n=10)$ y octoploide $(2 n=40)$; la meiosis del diploide es normal, forma 5 II en metafase I, mientras que el citotipo octoploide (cuadro IV) presenta octovalentes en $76 \%$ de las células (en la Fig. I 4 se observan 3 octovalentes, 2 tetravalentes y 4 bivalentes), por lo que se podría considerar como un autooctoploide.

En T. subulata, Arbo y Fernández (1983), encontraron que los tetraploides están más extendidos geográficamente; sobre 12 poblaciones estudiadas, 11 eran tetraploides, Barrett y Shore (1980) informan que en T. scabra sobre 7 poblaciones, 6 eran diploides; en esta comunicación se dan a conocer además 2 poblaciones diploides y una tetraploide. En T. Krapovickasii sobre 7 poblaciones, 4 son diploides y 3 tetraploides. En T. grandi- 
Cuadro IV. Promedio \pm E.S. y variación de las asociaciones cromosómicas en metafase I, en citotipo octoploide $(2 \mathrm{n}=$ 40) de T. grandiflora S. 21385 (Serie Canaligerae)

\begin{tabular}{llllllllc}
\hline I & II & III & IV & V & VI & VII & VIII & N.CMP \\
\hline 0,19 & 5,14 & 0,23 & 2,19 & 0,09 & 1,23 & 0,04 & 1,47 & 21 \\
$\pm 0,11$ & $\pm 0,48$ & $\pm 0,13$ & $\pm 0,41$ & $\pm 0,06$ & $\pm 0,25$ & $\pm 0,04$ & $\pm 0,22$ & \\
$(1-2)$ & $(1-10)$ & $(0-2)$ & $(0-6)$ & $(0-1)$ & $(0-4)$ & $(0-1)$ & $(0-3)$ &
\end{tabular}

flora se encontraron 6 poblaciones diploides y una octoploide.

Todas las especies estudiadas de la serie Canaligerae, salvo T. hermannioides y $T$. aff. coriacea, pertenecían al complejo T. ulmifolia, con categoría de variedad. Algunas de estas entidades han sido elevadas a la categoría de especie (Arbo, 1985), teniendo presente los caracteres morfológicos y los datos citológicos presentados en este trabajo, que apoyan y valorizan dicha separación. Por ejemplo $T$. grandidentata, $2 \mathrm{n}=4 \mathrm{x}=20$, tiene comportamiento meiótico regular, forma en todas las células 10 II, por lo que sería un alotetraploide, originado por hibridación de dos especies diferentes y posterior duplicación cromosómica, quedando constituído por dos genomios diferentes duplicados. La misma interpretación es válida para T. ulmifolia, T. orientalis $(2 \mathrm{n}=6 \mathrm{x}=30)$ y $T$. Aurelii $(2 \mathrm{n}=8 \mathrm{x}=40)$ que presentan 15 II y 20 I respectivamente en todas las células estudiadas. Estas tres últimas especies serían alohexaploides y alooctoploide respectivamente, aunque en $T$. Aurelii se observaron dos células con 18 $\Pi+1$ IV. Este tetravalente puede estar indicando una translocación recíproca o que sea un alooctoploide de índole segmentaria. Por lo expuesto anteriormente, estas entidades biológicas deben pertenecer a diferentes especies.

Es interesante señalar que en un híbrido, T. subulata $\times T$. scabra, $2 \mathrm{n}=10$, se encontraron en la misma planta, raíces secundarias con $2 n=10,20$ y 40 . Este hallazgo sumado al hecho de que es 
Cuadro V. Promedio \pm E.S. y variación de las asociaciones cromosómicas en metafase I, en subespecies de $T$. sidoides (serie Leiocarpae)

\begin{tabular}{llllllll}
\hline Subespecie & $2 \mathrm{n}$ & I & II & III & IV & VI & N \\
\hline & 14 & & 7 & & & & \\
integrifolia & 14 & & & & & &
\end{tabular}

A. 385

integrifolia

28

0,22

7,33

0,22

3,11

9

A. 2418

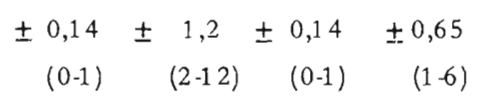

holosericea

28

0,28

10,6

0,09

1,5

21

A. 2461

$\pm 0,17$

(0-3)

$\pm 0,47 \pm 0,06 \pm 0,22$.

28

6,25

$(0-1) \quad(0-4)$

holosericea

A. 2525

$\pm \quad 1,25$

3,87

8

(2-12)

$\pm 0,61$

(1-6)

carnea

A. 2510

28

0,08

6,36

0,01

3,78

84

$\pm 0,04 \pm 0,30 \pm 0,01 \quad \pm 0,15$

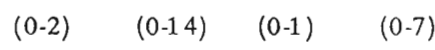

carnea

K. 25847

42

12,33

3,11

$0,44 \quad 9$

$\pm 1,59$

$\pm 0,63 \pm 0,17$

(4-18)

$(0-7)$

(0-1)

carnea

C. 1782

42

$$
0,75
$$

$$
7,87
$$

0,25

3,95

$1,75 \quad 8$

$\pm 0,41 \pm 1,12 \pm 0,16 \pm 0,59 \pm 0,45$

$(0-3)$

(3-12)

$(0-1)$

$(1-6)$

$(0-4)$

común observar en Turnera nuevas plantas a partir de raíces, hace pensar que podría ser uno de los mecanismos para originar autopoliploides. Probablemente el citotipo autooctoploide de $T$. grandiflora se haya originado a partir de una raíz secundaria con $2 n=8 x=40$. Hasta el presente en esta especie no se encontró ningún citotipo con nivel de ploidía intermedio entre el diploi. de y el octoploide. 


\section{Cuadro VI. Fertilidad de polen}

\begin{tabular}{|c|c|c|c|c|}
\hline Especie & Coleccionista & $\begin{array}{l}\text { Nivel de } \\
\text { ploidía }\end{array}$ & $\begin{array}{l}\text { \%de } \\
\text { fert. }\end{array}$ & $\begin{array}{l}\text { Rango de } \\
\text { variación }\end{array}$ \\
\hline
\end{tabular}

Ser ie Canaligerae, $\mathrm{x}=5$

T. scabra
T. scabra
T. subulata
T. surinamensis
T. Krapovickasii
T. Krapovickasii
T. grandiflora
T. grandiflora
T. Aurelii
T. oriertalis
T. grandidentata
T. aff. coriacea
T. hermannioides
T. caerulea

$\begin{array}{llll}\text { Ar. } 1354 & 2 \mathrm{x} & 94,57 & \\ \text { J. } 8769 & 4 \mathrm{x} & 73,9 & 48-92 \\ \text { K. } 37115 & 4 \mathrm{x} & 86,75 & 84-88 \\ \text { K. } 38751 & 2 \mathrm{x} & 57,83 & 38-81 \\ \text { Ah. } 4549 & 2 \mathrm{x} & 96,7 & 95-98 \\ \text { S. } 19514 & 4 \mathrm{x} & 87,7 & 74-94,3 \\ \text { F. } 366 & 2 \mathrm{x} & 95,85 & 92-99 \\ \text { S. } 21385 & 8 \mathrm{x} & 71,76 & 60-85 \\ \text { S. } 23860 & 8 \mathrm{x} & 96,25 & 94-98 \\ \text { A. } 1538 & 6 \mathrm{x} & 97 & 95-99 \\ \text { A. } 1674 & 4 \mathrm{x} & 93 & 90-96 \\ \text { K. } 38793 & 2 \mathrm{x} & 98,15 & 98-98,3 \\ \text { K. } 38729 & 2 \mathrm{x} & 88,5 & 72-97,5 \\ \text { K. } 38740 & 2 \mathrm{x} & 88 & 86-90\end{array}$

Seric Leiocarpae, $\mathrm{x}=7$

\begin{tabular}{|c|c|c|c|c|}
\hline T. melochioides & K. 38621 & $2 x$ & 93,3 & $91-95$ \\
\hline$T$ Hassleriana & A. 1735 & $4 \mathrm{x}$ & 59 & $32-74$ \\
\hline T. sidoides ssp. carnea & S. 21711 & $2 \mathrm{x}$ & 97,5 & $97-98$ \\
\hline$"$ & A. 2510 & $4 \mathrm{x}$ & 74 & $51-97$ \\
\hline T. sidoides ssp. integrifolia & A. 385 & $2 \mathrm{x}$ & 89 & \\
\hline$"$ & A. 2506 & $4 x$ & 62,5 & \\
\hline$"$ & A. 2418 & $4 \mathrm{x}$ & 45 & \\
\hline$"$ & A. 2487 & $6 x$ & 67,35 & $65-70,5$ \\
\hline$"$ & A. 2419 & $8 \mathrm{x}$ & 75 & $66-84$ \\
\hline T. sidoides ssp. holosericea & A. 2513 & $4 \mathrm{x}$ & 94,5 & 92.97 \\
\hline ," & A. 2478 & $4 \mathrm{x}$ & 53,8 & $53,6-54$ \\
\hline$"$ & A. 2495 & $6 \mathrm{x}$ & 87 & \\
\hline T. sidoides ssp. pinnat if ida & S. 21648 & $4 \mathrm{x}$ & 90 & $88-92$ \\
\hline
\end{tabular}

La fertilidad de polen en los alopoliploides (cuadro VI) es elevada como cabría esperar, superior a 93\%. Los autotetraploides tienen una fertilidad también alta, desde $59 \%$ en $T$. Hassleriana $2 \mathrm{n}=4 \mathrm{x}=28$ hasta $94,5 \%$ en $T$. sidoides ssp. holoseri- 
cea $2 n=4 x=28$. Entre los autohexaploides la fertilidad de polen varía desde $67,35 \%$ en $T$. sidoides ssp. integrifolia $2 \mathrm{n}=6 \mathrm{x}=42$ y $87 \%$ en $T$. sidoides ssp. holosericea $2 \mathrm{n}=6 \mathrm{x}=42$. En el autooctoploide $T$. sidoides ssp. integrifolia $2 \mathrm{n}=8 \mathrm{x}=56$, la fertilidad es de $75 \%$ y en $T$. grandiflora citotipo octoploide $2 n=8 x=40$ es de $71,6 \%$. Estos valores son aparentemente altos por tratarse de autopoliploides, pero se podría explicar, porque la mayoría de las gametas probablemente sean fértiles al tener uno o más genomios completos, aún con algunos de los cromosomas repetidos (aneuploides). Sin embargo en la competencia por la fecundación tendrían mayor posibilidad de éxito las gametas euploides que las aneuploides.

Cuadro VII. Niveles de ploidía én poblaciones de T. sidoides, $\mathrm{x}=7$.

\begin{tabular}{lccccr}
\hline Subespecie & $2 \mathrm{x}$ & $4 \mathrm{x}$ & $5 \mathrm{x}$ & $6 \mathrm{x}$ & $8 \mathrm{x}$ \\
\hline sidoides & & & & & \\
holosericea & & 1 & $1 ?$ & & \\
carnea & 1 & 1 & & 2 & \\
integrifolia & 1 & 2 & & 2 & 1 \\
pinnatifida & & 2 & & & \\
Total & & & & & \\
$\%$ & 2 & 10 & 1 & 6 & 1 \\
& 10 & 50 & 5 & 30 & 5
\end{tabular}

Lewis (1967) analizó 135 plantas derivadas de autotriploiảes de Delphinium, y solamente encontró un aneuploide estéril. Arbo y Fernández (1983), encontraron dos plantas diploides descendientes de un triploide polinizado por diploide en T. subulata. En los casos que las gametas aneuploides tuvieran éxito en la fecundación, las semillas resultantes, germinan, pero no progresan más allá del estado de plántulas, como se pudo comprobar en T. sidoides ssp. sidoides, Arbo 2435 (cuadro I). 


\section{Piriqueta}

En este género, las cuatro especies previamente estudiadas tienen 2n=14, $P$. glabrescens, $P$. tomentosa (Lewis et al., 1962), $P$. cistoides y $P$. caroliniana (Ornduff, 1970). En este trabajo se determinó el mismo número cromosómico en $F$. aff. duarteana (Fig. 8), $P$. viscosa, P. serrulata y $P$. racemosa. Fn esta última especie se encontraron raíces con $2 n=14$ y $2 n=28$. Hasta el presente son 8 especies diploides, 2 tetraploides: $P$. rosea y $P$. sujorbicularis y una especie hexaploide, $P$. taubatensis con $2 n=42$.

\section{Conclusiones}

La alo y autopoliploidía tuvieron un rol importante en la evolución de las especies de Turnera y Piriqueta. El 27\% de las especies de Turnera con el número básico $\mathrm{x}=7$ son poliploides. Una de estas especies, $T$. sidoides tiene 5 subespecies, cada una de las cuales está representada con distintos niveles de ploidía; en 20 poblaciones estudiadas, se encontraron únicamente $10 \%$ de diploides, $50 \%$ de tetraploides, $30 \%$ de hexaploides y $5 \%$ de octoploides (cuadro VII); todos estos poliploides se comportan citológicamente como autopoliploides. En la serie Canaligerae con el número básico $\mathrm{x}=5$, el $33 \%$ de las especies está representada por citotipo diploide yautotetraploide yel $33 \%$ por alopoliploides. El género Piriqueta posee es número básico $\mathrm{x}=7 \mathrm{v}$ el $25 \%$ de las especies son poliploides.

En este trabajo se estudiaron 6 series de las 9 que posee el género Turnera (Urban, 1883): En 4 series el número básico es $\mathrm{x}=7$ (Salicifoliae, Stenodictyae, Leiocarpae y Microphyllae); mientras que en Canaligerae es $\mathrm{x}=5$. Papilliferae, es una serie monotípica, $T$. chamaedryfolia con $2 \mathrm{n}=26$, por lo que presentaría un número básico derivado, $\mathrm{x}=13$, por aneuploidía de una especie con $2 n=4 x=28$. Con estos datos se podría aventurar 
que el número básico más primitivo del género Turnera sería $x=7$. A partir de éste se habrían originado las especies con $x=5$ $\mathrm{y} x=13$.

\section{Agradecimiento}

Deseo expresar mi agradecimiento a la Licenciada María M. Arbo, por la gentileza de haberme facilitado las semillas, por la determinación del material estudiado y por la lectura del manuscrito.

\section{Bibliografía}

Arbo, M.M. 1977. Adenoa, nuevo género americano de Turneraceae. Hickenia. 1 (16): 87-92.

Arbo, M.M. 1979. Revisión del género Erblichia (Turneraceae). Adansonia ser. 2, 18(4): 459-482.

Arbo, M.M. 1985. Notas taxonómicas sobre Turneráceas sudamericanas. Candollea 40: 175-191.

Arbo, M.M. 1986. Una nueva Turnera del Paraguay. Candollea 41: 209-211.

Arbo, M.M. y A. Fernández. 1983. Posición taxonómica, citología y palinología de tres niveles de ploidía de Turnera subulata Smith. Bonplandia 5(23): 21 2-226.

Barrett, S.C.H. 1978. Heterostyly in a tropical weed: the reproductive biology of the Turnera ulmifolia complex (Turneraceae). Canadian J. Bot. 56(15): 1713-1725.

Barrett, S.C.H. and Shore. 1980. Variation in breeding systems in the Turnera ulmifolia complex. Trabajo presentado en el Second International Congress of Systematic and Evolutionary Biology, Vancouver, Canadá. 
Hamel, J.L. 1965. Le noyau et les chromosomes somatiques de Turnera ulmifolia L. Mém. Mus. Natl. His. Naî. Sér. B, Bot. 16(1): 3-8.

Lewis, H. 1967. The taxonomic significance of autopolyploidy. Taxon 16: 267-271.

Lewis, H.; C. Epling, G.A.L. Mehlquist and C.G. Wyckoff. 1951. Chromosome numbers of Californian Delphinium and their geographical occurrence. Ann. Missouri Bot. Gard. 38: $101-107$.

Lewis, W.H., H.L. Stripling and R.G. Ross. 1962. Chromosome numbers for some Angiosperms of the southern United States and Mexico. Rhodora 64,(758): 147-161.

Mroginski, L.A. y A. Fernández. 1979. Cultivo in-vitro ce anteras de especies de Arachis (Leguminosae). Oléagineux $3(5): 243-248$.

Ornduff, R. 1970. Relationships in the Piriqueta carolinianaP. cistoides complex (Tumeraceae). J. Arnold Arbor. 5: $491-498$.

Raman, V.S. and P.C. Kesavan. 1964. Meiosis and the nature of polyploidy in Turnera ulmifolia. J. Indian Bot. Soc. 43: 495-497.

Stebbins, G.L. 1951. Variation and evolution in plants. 2nd. ed. Columbia Univ. Press. New York.

Urban, I. 1883. Monographie der Familie der Turneraceen. Jahrb. Königl. Bot. Gart. Berlin 2: 1-152. 